\section{Assessing sedation need and managing referred dentally anxious patients: is there a role for the Index of Sedation Need?}

\author{
S. Yuan, ${ }_{1}^{* 1}$ S. J. Carson, ${ }_{1}^{1}$ M. Rooksby, ${ }_{1}^{1}$ J. McKerrow, ${ }_{1}^{2}$ C. Lush ${ }^{2}$ and \\ R. Freeman ${ }^{1,3}$
}

VERIFIABLE CPD PAPER
IN BRIEF

- Discusses the use of the IOSN for general dental practitioners regarding the appropriate referral criteria to specialist services for patients with dental anxiety.

- Provides a method by which dentists can reliably assess and tailor the sedation modality for patients with dental anxiety.

- Shows the importance of assessing patient dental anxiety and treatment complexity in the decision making process.

\begin{abstract}
Aim To conduct an exploratory investigation of public dental service (PDS) practitioners' planned sedation modality using a structural equation modelling approach, in order to identify the explanatory value of using the Index of Sedation Need (IOSN), or its component parts, to predict sedation modality in patients referred with dental anxiety. Methods $\mathrm{A}$ convenience sample of patients referred to the PDS for dental anxiety management was invited to take part. The IOSN was completed for each patient (patient dental anxiety, medical and behavioural indicators and dental treatment complexity) as well as the American Society of Anesthesiologists Physical Status Classification System and the Case Mix Tool. The practitioners completed details of their planned sedation modality and identified normative dental treatment need. The data were entered onto an SPSS v21 database and subjected to frequency distributions, t-tests, correlation analysis and exploratory partial structural equation modelling (SEM). Results Ninety-five percent of patients were ranked as MDAS 3 or 4, indicating high dental anxiety; $69 \%$ had a medical condition, which might impact on dental treatment and 82\% had a dental treatment need, which was classified as intermediate/complex according to the IOSN. Eighty-eight percent of the patients in accordance with the IOSN required sedation: 62\% of patients were assessed as requiring intravenous sedation. The IOSN discriminated between patients who were assessed as requiring more complex sedation modalities and had a greater normative treatment need. The SEM showed that the patient dental anxiety $(P<0.02)$ and dental treatment complexity $(P<0.02)$ predicted planned sedation modality. Functional morbidity was less strong, as a predictor, and was significant at the ten percent level. Conclusions The IOSN is a useful and valid assessment of sedation need and predicted sedation modality for patients referred with high dental anxiety states and secondly, that component parts of the IOSN add explanatory value in practitioners' choice of planned sedation modality.
\end{abstract}

\section{INTRODUCTION}

The recent intercollegiate report on Standards for conscious sedation in the provision of dental care ${ }^{1}$ highlights the importance of using appropriate audit tools to review clinical outcomes of sedation services in dental care, in order to ensure good clinical practice. A central strand of ensuring good practice is the identification of the type of sedation required through rigorous assessment of the patient. An emphasis is placed upon the thoroughness of the assessment

Dental Health Services Research Unit, University of Dundee, DD1 4HN, ${ }^{2}$ Public Dental Service, NHS Highland, IV2 7GE; ${ }^{3}$ Dental Public Health, NHS Tayside

${ }^{*}$ Correspondence to: Dr S Yuan

Email:s.z.yuan@dundee.ac.uk

\section{Refereed Paper}

Accepted 19 October 2015

DOI: $10.1038 /$ sj.bdj.2015.955

${ }^{\circ}$ British Dental Journal 2015; 219: 571-576 with the decision to provide conscious sedation being, 'based on a full assessment in respect of healthcare history, psychological needs and overall management.' ${ }^{1}$ It was apparent that the need for a valid and reliable assessment of sedation need was necessary if adherence to the Royal Colleges' recommendation for good practice was to be obtained, with regard to the assessment of the patient.

In 2011, Coulthard and colleagues ${ }^{2-6}$ developed the IOSN as a means of identifying, assessing and delivering tailored sedation to patients. The underlying premise of the IOSN was that patients requiring sedation were not simply dentally anxious, but that physical and dental treatment complexity issues should also be considered. The IOSN was, therefore, composed of three main elements: [i] the Modified Dental Anxiety Scale (MDAS); [ii] Medical and Behavioural
Indicators; and [iii] Dental Treatment Complexity. Total scores ranged from 3-11 with cut-offs at 4 indicating minimal sedation need; at 6 indicating moderate sedation need; at 9 indicating high sedation need and scores between 10-11 indicating very high sedation need. The developers ${ }^{2-6}$ stated that IOSN could be used as a referral tool (as above) or as a health needs assessment device for practitioners. ${ }^{2-7}$ In this latter role, scores between 3-6 indicate no sedation need, whereas scores between 7-11 indicate sedation need. Early work on the use of the IOSN was encouraging and suggested that the IOSN was a better indicator of sedation need than relying on dental anxiety prevalence alone. ${ }^{4,5}$ More recently Liu et al. ${ }^{7}$ examined the predictive validity of the IOSN together with measures of its specificity and sensitivity. They showed that in patients self-referred to an NHS oral surgery 
referral service, two components of the IOSN, namely the Modified Dental Anxiety Scale and Dental Treatment Complexity predicted the likelihood of receiving or not receiving sedation. This work suggested that the IOSN had construct validity since it differentiated between those receiving local anaesthesia, intravenous sedation (IV) or dental general anaesthesia (DGA).

Therefore, the IOSN provided an assessment of sedation need, built upon the components of dental anxiety, physical morbidity and dental treatment complexity. Moreover, it is suggested that, in addition, the IOSN had a degree of predictive and construct validity with regard to patients attending an NHS oral surgery service. ${ }^{7}$ The question remained, however, as to whether the IOSN and its component parts, could predict, or assist in the prediction of the type, or modality of sedation required for the patient referred with dental anxiety. As part of their on-going sedation audit procedures, PDS, NHS Highland became interested to know the additional value of using the IOSN in conjunction or instead of their current assessment system for patients referred for dental anxiety management to the PDS sedation service. The PDS dentists' assessment included, requesting the patients to complete the MDAS, the completion of the American Society of Anesthesiologists Physical Status Classification System ${ }^{8}$ and the Case Mixed Tool ${ }^{9,10}$ and an assessment of the normative treatment need. Therefore the aim was to conduct an exploratory investigation of practitioners' planned sedation modality using a structural equation modelling approach in order to identify the explanatory value of using IOSN or its component parts to predict sedation modality in patients referred with dental anxiety.

\section{METHOD}

\section{Sample}

A convenience sample of 100 adult dental patients referred from general dental practitioners to the 14 PDS clinics, over a 12-month period, for dental anxiety management, were invited to take part. It was estimated that a sample size of 100 patients would be required to participate, in order to show a statistically reliable difference (at 90\% power, at $\mathrm{P}<0.001$, two-tailed) in the level of sedation need compared to the rest of the population who are not dentally anxious based on a mean MDAS score of $10.65( \pm 5.55)$.

All participants were provided with a participant information sheet with their dental appointment and were asked to consent to participate. Consent was obtained at the appointment visit by the assessing PDS dentist.

\section{Questionnaires}

\section{Patient questionnaire and administration}

\section{Modified Dental Anxiety Scale} (MDAS): ${ }^{11,12}$ the patient questionnaire was MDAS using a five-item inventory to assess anticipatory dental anxiety associated with waiting for treatment, waiting for the drill, scale and polish and the local anaesthesia. The questions are of a 5-point Likert scale format ranging from 1 (not anxious) to 5 (extremely anxious). The possible range of raw scores in 5-25, with scores over 19 indicative of dental phobia

2. Administration of MDAS: the MDAS is routinely used as a patient assessment of dental anxiety in the referral PDS clinics. Therefore, following the usual procedure, participants were requested to complete the questionnaire while waiting for their dental assessment appointment.

\section{PDS practitioner assessment ques- tionnaire of patient dental anxiety management}

The PDS practitioner assessment questionnaire was in two parts. The first part was the IOSN. The second part included two routinely recorded health (American Society of Anesthesiologists [ASA] Physical Status Classification System ${ }^{8}$ ) and dental indicators (Case Mixed Tool) as well as the MDAS, which, as stated previously, was routinely completed by the patient. The patient's ASA Classification Category and Case Mix Tool Score were used as markers for comparison with IOSN. In addition, the practitioner was asked to note the type of dental anxiety management they had prescribed for the patient, their planned dental treatment and the patient's age and gender.

Part 1: Index of Sedation Need (IOSN) $)^{2-6}$ The IOSN is a 3-component instrument to assess the need for conscious sedation and consists of:

\section{Modified Dental Anxiety Scale}

(MDAS): the raw MDAS total scores are converted into rank scores with a score of 1 ('minimal anxiety') equivalent to total MDAS score of 5-9; a score of 2 ('moderate anxiety') equivalent to total MDAS scores of 10-12; a score of 3 ('high anxiety') equivalent to total MDAS scores of 13-17; and a score of 4 ('very high anxiety') equivalent to total MDAS scores of 18-25

2. Medical and Behavioural Indication Score (MBIS): MBIS is assessed on a 4 -item checklist. This checklist ranged

\section{Table 1 Current definitions and examples of ASA classification ${ }^{8}$}

\begin{tabular}{|c|c|c|}
\hline $\begin{array}{l}\text { ASA } \\
\text { classification }\end{array}$ & Definition & Examples, including, but not limited to: \\
\hline ASA I & A normal healthy patient & Healthy, non-smoking, no or minimal alcohol use \\
\hline ASA II & A patient with mild systemic disease & $\begin{array}{l}\text { Mild diseases only without substantive functional limitations. Examples include (but not limited to): current } \\
\text { smoker, social alcohol drinker, pregnancy, obesity }(30<\mathrm{BMI}<40) \text {, well-controlled DM/HTN, mild lung disease }\end{array}$ \\
\hline ASA III & $\begin{array}{l}\text { A patient with severe systemic } \\
\text { disease }\end{array}$ & $\begin{array}{l}\text { Substantive functional limitations; } \\
\text { One or more moderate to severe diseases. Examples include (but not limited to): poorly controlled DM or HTN, } \\
\text { COPD, morbid obesity (BMI } \geq 40 \text { ), active hepatitis, alcohol dependence or abuse, implanted pacemaker, moder- } \\
\text { ate reduction of ejection fraction, ESRD undergoing regularly scheduled dialysis, premature infant PCA <60 } \\
\text { weeks, history ( }>3 \text { months) of MI, CVA, TIA, or CAD/stents. }\end{array}$ \\
\hline ASA IV & $\begin{array}{l}\text { A patient with severe systemic dis- } \\
\text { ease that is a constant threat to life }\end{array}$ & $\begin{array}{l}\text { Examples include (but not limited to): recent ( }<3 \text { months) MI, CVA, TIA, or CAD/stents, ongoing cardiac } \\
\text { ischemia or severe valve dysfunction, severe reduction of ejection fraction, sepsis, DIC, ARD or ESRD not } \\
\text { undergoing regularly scheduled dialysis }\end{array}$ \\
\hline ASA V & $\begin{array}{l}\text { A moribund patient who is not } \\
\text { expected to survive without the } \\
\text { operation }\end{array}$ & $\begin{array}{l}\text { Examples include (but not limited to): ruptured abdominal/thoracic aneurysm, massive trauma, intracranial } \\
\text { bleed with mass effect, ischemic bowel in the face of significant cardiac pathology or multiple organ/system } \\
\text { dysfunction }\end{array}$ \\
\hline ASA VI & A declared brain-dead patient whose & jans are being removed for donor purposes \\
\hline
\end{tabular}


from 'no medical/behavioural indicators', scoring 1; 'systematic disorders (not of severity to exclude sedation)', scoring 2; 'systematic disorders that compromise ability to cooperate', scoring 3; and 'gag reflex' scoring 4

3. Dental Treatment Complexity Score (DTCS): DTCS is assessed on a 4-point checklist. The items include, routine treatment (for example, single quadrant restorations) scoring 1 ; intermediate treatment (for example, multi-tooth extraction), scoring 2; complex treatment (for example, surgical extraction with bone removal) scoring 3; and highly complex treatment (for example, multiples of complex treatments) scoring 4 . The scores were derived from the patient's planned dental treatment, as stated below.

The above 3 individual sedation need component scores are summed together to give a total IOSN score. Possible scores range from 3 to 12 , with scores of 3-6 specifying no or minimal sedation need, scores between 5-6 designating a moderate sedation need, and scores above 7 indicating a high (scores 7-9) or very high (scores 10-12) sedation need.

Part 2: routine indicators

1. American Society of Anesthesiologists Physical Status Classification System (ASA): ${ }^{8}$ Table 1 shows the classification and descriptors of the ASA classification for patients being assessed for sedation for dental anxiety management

2. Simplified Case Mixed Tool (sCMT): ${ }^{10}$ The Weighted Case Mix Tool (WCMT) was originally devised by Bateman and colleagues. ${ }^{9}$ The WCMT assessed the complexity of the provision of care for disabled people through a structured matrix. The criteria include: ability to communicate, ability to cooperate, medical status, oral risk factors, access to oral care, and legal and ethical barriers to care. These criteria are evaluated with a four-point scale from 0 which denotes an average 'fit and well' individual and $\mathrm{A}, \mathrm{B}, \mathrm{C}$ which indicate increasing levels of complexity.

The Simplified Case Mix Tool ${ }^{10}$ (sCMT) is a modification of Bateman's original Weight Case Mixed Tool. ${ }^{9}$ It omits the oral risk factor and thus has five criteria. For this analysis the decision was made to adopt the scoring criteria as defined sCMT because the scoring criteria are of: 1) higher level of reliability; 2) have better discrimination between reference groups; and 3) there is ease of interpretations. ${ }^{10}$ Therefore, in accordance with the sCMT, the original WCMT was collapsed to a three-point scale where 0 represents 'fit and well individual (scored as 0), A represents 'moderate' level of complexity (scored as 1), and B/C indicates 'severe' level of complexity (scored as 2).

1. Planned sedation modality: practitioners were asked to note the type of dental anxiety management they had prescribed for the patient - whether behavioural management (scoring 0), inhalation sedation (scoring 1), intravenous sedation (scoring 2) or dental general anaesthesia (scoring 3). The prescription was based upon their clinical examination, and psychological assessment of the patient

2. Normative dental treatment need: the practitioners were asked to note the planned dental treatment using a checklist, which included dental examination, restorations, periodontal treatment, extractions and so forth. Each time a treatment item was checked a score of 1 was awarded. The normative dental treatment need was converted into Dental Treatment Complexity Scores (DTCS) in accordance with the agreed criteria of the originators of the IOSN measure

3. Administration of assessment questionnaire: the participating dental practitioners completed the MBIS and DTC parts of the IOSN at the patient's sedation assessment appointment, as well as the ASA categorisations, sCMT. The patients' age and gender were noted as well as their MDAS scores.

\section{Statistical analysis}

The data were entered onto an SPSS v21 database. The data were subjected to frequency distributions, t-tests, correlation analysis and exploratory partial structural equation modelling (AMOS V21).

\section{Ethical considerations}

The North of Scotland Research Ethics Committee was sent the protocol, participant information sheet and consent form. However, in their opinion, NHS ethical approval was not deemed necessary as the committee classified the study as audit.

\section{RESULTS}

\section{Sample}

Ninety-four patients who were referred to the PDS in the 12-month period for dental anxiety management assessment agreed to participate. One questionnaire was omitted due to missing information for the MBIS and TCS. Of the 93 patients with full data sets, 23\% were male. The age of the participants ranged from 17 years to 71 years with a mean age of 39.96 years (95\% CI: 37.10, 42.80).
PDS practitioner assessment questionnaire: Part 1 IOSN

The results of the IOSN's three sedation need components: Modified Dental Anxiety Scale (MDAS) as raw and rank scores, Medical and Behavioural Indicator Score (MBIS), and Treatment Complexity Score (TCS) are presented.

1. MDAS: The MDAS total scores ranged from 7 to 25 with the mean of 21.13 (95\%CI: 20.33, 21.93). The highest mean scores were for the individual MDAS items of 'teeth drilled' (4.66 [95\%CI: $4.50,4.81])$ and 'local anaesthetic injection' (4.57 [95\%CI: 4.38, 4.76]). Eighty-three percent (77) of participants scored 19 or over which is indicative of dental phobia. For the individual MDAS items, 79\% (73) were extremely anxious about drilling and 77\% (72) about the prospect of having a local anaesthetic injection; 53\% (49) about visiting the dentist tomorrow and 29\% (27) were extremely anxious about having a scale and polish. The MDAS total raw scores were converted to rank scores ranging from 1 (MDAS 5-9) to 4 (MDAS 18-25) in accordance with scoring for IOSN. ${ }^{2-3}$ The rank scores indicated that 95\% (88) were ranked as 3 or 4 and were designated as having high or very high dental anxiety (Table 2)

2. MBIS: Thirty-one percent (29), of participants referred for dental anxiety management assessment were assessed as having no medical or behavioural indicators that could affect dental treatment. The majority of participants (56\%) were assessed as having a medical condition, which could be exacerbated by dental treatment and a further 13\% of participants were assessed as having a systematic illness that could affect cooperation and the provision of dental treatment (Table 2)

3. DTCS: Eighteen percent (17) of patients were assessed as requiring routine treatment: $82 \%$ of patients were assessed needing treatments of intermediate level or complex level. None of the referred patients needed highly complex treatment (Table 2)

4. Total IOSN Scores: The total scores of the three sedation need domains were summed together to give a total IOSN score and to provide a sedation need category. Seventy-nine (85\%) of the referred patients had total IOSN scores of between 7-9, indicating a high sedation need. A further two patients had total IOSN scores of between 10-12 and were categorised as having a very high need for sedation (Table 2). 


\begin{tabular}{|c|c|c|c|c|c|c|c|c|c|c|}
\hline $\begin{array}{l}\text { [i] MDAS } \\
\text { Raw scores }\end{array}$ & $\begin{array}{l}\text { Rank } \\
\text { score }\end{array}$ & $\begin{array}{l}\mathrm{n} \\
(\%)\end{array}$ & $\begin{array}{l}\text { [ii] Medical and Behavioural } \\
\text { Indicator items (MBIS) }\end{array}$ & $\begin{array}{l}\text { Rank } \\
\text { score }\end{array}$ & $\begin{array}{l}n \\
(\%)\end{array}$ & $\begin{array}{l}\text { [iii] Indicators of treatment } \\
\text { complexity (DTCS) }\end{array}$ & $\begin{array}{l}\text { Rank } \\
\text { score }\end{array}$ & $\begin{array}{l}\mathrm{n} \\
(\%)\end{array}$ & $\begin{array}{l}\text { Total IOSN scores \& } \\
\text { descriptors }\end{array}$ & $\begin{array}{l}n \\
(\%)\end{array}$ \\
\hline $5-9$ & 1 & $\begin{array}{l}2 \\
(2)\end{array}$ & $\begin{array}{l}\text { No medical or behavioural } \\
\text { indicators }\end{array}$ & 1 & $\begin{array}{l}29 \\
(31)\end{array}$ & $\begin{array}{l}\text { Routine - Scale, single rooted extrac- } \\
\text { tion of } 1 \text { or } 2 \text { teeth, small soft tissue } \\
\text { biopsy, single quadrant restorations, } \\
\text { crown preparations or anterior endo- } \\
\text { dontic treatment }\end{array}$ & 1 & $\begin{array}{l}17 \\
(18)\end{array}$ & $\begin{array}{l}\text { Minimal need for } \\
\text { sedation } \\
\text { Total IOSN scores 3-4 }\end{array}$ & $\begin{array}{l}1 \\
(1)\end{array}$ \\
\hline $10-12$ & 2 & $\begin{array}{l}3 \\
(3)\end{array}$ & $\begin{array}{l}\text { Systemic disorders that may be } \\
\text { exacerbated by treatment: eg, } \\
\text { fainting attacks/hypertension/ } \\
\text { angina/asthma/epilepsy/other }\end{array}$ & 2 & $\begin{array}{l}52 \\
(56)\end{array}$ & $\begin{array}{l}\text { Intermediate - Scale and root plan- } \\
\text { ning multi-rooted tooth extraction, } \\
\text { surgical extraction without bone } \\
\text { removal, apicectomy anterior tooth, } \\
2 \text { quadrant restorative, posterior } \\
\text { endodontic treatment }\end{array}$ & 2 & $\begin{array}{l}52 \\
(56)\end{array}$ & $\begin{array}{l}\text { Moderate need for } \\
\text { sedation } \\
\text { Total IOSN scores 5-6 }\end{array}$ & $\begin{array}{l}11 \\
(12)\end{array}$ \\
\hline $13-17$ & 3 & $\begin{array}{l}10 \\
(11)\end{array}$ & $\begin{array}{l}\text { Systemic disorders that compro- } \\
\text { mise ability to cooperate: eg, } \\
\text { arthritis/Parkinsonism/multiple } \\
\text { sclerosis/other }\end{array}$ & 3 & $\begin{array}{l}12 \\
(13)\end{array}$ & $\begin{array}{l}\text { Complex-Periodontal surgery, surgi- } \\
\text { cal extraction with bone removal, } \\
\text { apicectomy posterior tooth, multiple } \\
\text { quadrant restorative, multiple poste- } \\
\text { rior endodontics }\end{array}$ & 3 & $\begin{array}{l}24 \\
(26)\end{array}$ & $\begin{array}{l}\text { High need for sedation } \\
\text { Total IOSN scores 7-9 }\end{array}$ & \begin{tabular}{|l}
79 \\
$(85)$
\end{tabular} \\
\hline $18-25$ & 4 & $\begin{array}{l}78 \\
\text { (84) }\end{array}$ & Gag reflex & 4 & - & $\begin{array}{l}\text { Highly complex -Any treatment } \\
\text { considered more complex than above } \\
\text { or are multiples of the above }\end{array}$ & 4 & - & $\begin{array}{l}\text { Very high need for } \\
\text { sedation } \\
\text { Total IOSN scores 10-12 }\end{array}$ & $\begin{array}{l}2 \\
(2)\end{array}$ \\
\hline
\end{tabular}

Table 3 Comparison of MDAS, ASA, sCMT scores, planned sedation modality and normative dental treatment need scores by IOSN categorisation: health needs assessment

\begin{tabular}{|c|c|c|c|c|c|}
\hline \multirow{3}{*}{\multicolumn{2}{|c|}{$\begin{array}{l}\text { Routine patient indicators and } \\
\text { planned sedation and treatment }\end{array}$}} & IOSN categorisation: health needs assessment & IOSN categorisation: health needs assessment & \multirow{3}{*}{$t$} & \multirow{3}{*}{$P$} \\
\hline & & \multirow{2}{*}{$\begin{array}{l}\text { No sedation need } \\
\text { Mean }(95 \% \mathrm{CI})\end{array}$} & Yes sedation need & & \\
\hline & & & Mean $(95 \% \mathrm{Cl})$ & & \\
\hline \multirow{3}{*}{$\begin{array}{l}\text { Routine patient } \\
\text { indicators }\end{array}$} & MDAS score & $17.00(13.04,20.96)$ & $21.73(21.06,22.39)$ & -2.84 & $<0.001$ \\
\hline & ASA score & $0.50(0.17,0.83)$ & $0.80(0.68,0.92)$ & -1.77 & 0.08 \\
\hline & sCMT score & $2.08(1.58,2.59)$ & $2.58(2.39,2.76)$ & -1.91 & 0.06 \\
\hline \multirow{2}{*}{$\begin{array}{l}\text { Sedation and } \\
\text { dental treat- } \\
\text { ment need }\end{array}$} & $\begin{array}{l}\text { Planned sedation } \\
\text { modality score }\end{array}$ & $1.33(0.84,1.83)$ & $1.80(1.65,1.95)$ & -2.25 & 0.03 \\
\hline & $\begin{array}{l}\text { Normative dental } \\
\text { treatment need score }\end{array}$ & $1.92(1.18,2.66)$ & $3.08(2.78,3.37)$ & -2.84 & 0.006 \\
\hline
\end{tabular}

\section{PDS practitioner assessment question- naire: Part 2 Routine Indicators}

1. ASA: sixty-three percent (59) were classified as ASA II, that is, they had a mild systemic disease. Only six people were classified as ASA III with severe systemic disease

2. sCMT: the total mean score for sCMT was $2.52( \pm 0.85)$ with a range from 0 (no complexity) to 5 (severe complexity). The majority of the participants were assessed as having a moderate case mixed complexity with scores of $2(45 \%)$ and $3(40 \%)$. Only nine participants were assessed as having a severe case mixed complexity with scores of $4(9 \%)$ and $5(1 \%)$

3. Planned sedation modality: Twentytwo patients (24\%) were assessed by practitioners as requiring inhalation sedation, and a further 58 (62\%) participants were assessed as requiring intravenous sedation. Eight patients required general anaesthesia and five patients were assessed as requiring only behavioural management. The mean planned sedation modality score was $1.74( \pm 0.69)$

4. Normative dental treatment need: eighty-four percent (79) were assessed as needing restorative treatment, including dentures (14), fillings (59) and other procedures such as crowns and bridges (6). Sixty-five percent (60) required periodontal treatment and 80\% (74) required extractions. The mean planned treatment score was $2.94( \pm 1.34)$.

\section{IOSN, routine patient indicators and planned sedation modality}

Using the IOSN matrix, participants with IOSN scores between 3-6 were categorised as having minimal/moderate sedation need and those with scores between 7-11 were categorised as a high/very high sedation need. According to the IOSN Health Needs Assessment classification ${ }^{2,3}$ 12 patients, therefore, did not have a sedation need and 81 patients did have a sedation need.
Patients who were classified as needing sedation (21.73 [95\% CI: 21.06, 22.39]) had statistically significantly higher mean MDAS scores compared with those who were classified as not needing sedation (17.00 [95\% CI: 13.04, 20.96]) ( $\mathrm{P}<0.001)$. Patients who were classified as needing sedation compared with those who were classified as not needing sedation had statistically significantly higher mean scores for planned sedation modality and dental treatment need. There was a difference in mean scores for ASA $(\mathrm{P}=0.06)$ and sCMT $(\mathrm{P}=0.08)$ between the sedation need groups (Table 3 ).

The planned sedation modality was carefully examined and assessed as a single rating ranging from no sedation or behavioural dental anxiety management to dental general anaesthetic with intervening categories on sedation in rank order of severity. The planned sedation modality variable was promoted to interval scaling for the purposes of the structural equation modelling analysis. There were three major explanatory variables specified. of these, two were specified as latent variables, 
namely, functional morbidity and dental anxiety. Latent variables were constructed where possible to reduce the influence of measurement error. Functional morbidity was defined by three indicator variables including MBIS, ASA and the sCMT. Dental anxiety was defined by the five item rating scales that constitute the Modified Dental Anxiety Scale (MDAS). The third major explanatory variable was Dental Treatment Complexity Score (DTCS). This was derived from the practitioners' categorical description of the normative dental treatment need and was converted to be consistent with the IOSN categories for dental treatment complexity (DTC). The values were placed into a rank order of complexity according to the agreed criteria of the originators of the IOSN measure.

A simple non-mediational model was designed for testing against the raw data, that is, the three variables: functional morbidity (MBIS, ASA and sCMT), dental anxiety and DTC were directly implicated in explaining severity of sedation required by the patient. All three independent variables were allowed to correlate so that the direct paths to the dependent variable expressed the unique explanation taking into account the association with the other independent variables (Table 4).

The correlation matrix of the variables, including means and standard deviations, used in the modelling analysis was inspected. All coefficients showed sufficient independent variation without a large degree of overlap. The model was run with AMOS version 21, using maximum likelihood estimation and less than eight iterations were run to obtain convergence. The solution reported showed 'close fit' of the raw data to the specified model according to a number of pre-specified fit indices. All errors were independent with the exception of two correlated errors in the dental anxiety scale between anxiety about extraction and the other two treatment procedures. When these adjustments to the measurement model were included, it was found that the pattern of statistical associations was quickly iterated to a simple solution. The results are summarised in Figure 1. They show that three essential constructs (key factors) contribute to explaining the planned sedation modality. The diagram demonstrates that the indicators/ items are weighted descriptors of these constructs (for example, DTCS1 to DTCS4). The coefficients labelling the arrows that link the constructs to planned sedation modality are standardised and equivalent to correlations. The level of fit of this model was inspected by referring to the Chi-square value which was 31.1 , with 29 degrees of freedom. This confirmed that the probability of obtaining this result was two out of five times, which was regarded as not significantly different between the sampled raw data and specified model. Additional fit indices confirmed this elegant model description with the Comparative Fit Index (CFI) coming close to unity (0.99) and the root mean squared error of approximation (RMSEA) value at 0.03. Inspection of the factor loadings $(>0.4)$ of both latent variables showed significant loadings between the indicators and latent variables $(\mathrm{P}<0.001)$. The covariances between the independent variables were nonsignificant. The direct effects of two of the independent variables, namely, dental anxiety (MDAS) and treatment complexity, (DTCS) were each statistically significant $(\mathrm{P}<$ 0.02). The effect of functional morbidity was less strong, but significant at the ten percent level. When this path was constrained to zero in a further model testing the comparison between the original and constrained model, it remained at the same level $(\mathrm{P}<0.10)$ (Fig. 1).

\section{DISCUSSION}

With the publication of the 2015 Standards for conscious sedation ${ }^{1}$ and its predecessor published in 2007, ${ }^{13}$ the patient assessment was at the centre of providing a patient-centred

\begin{tabular}{l|l|l|l|l|l|l}
\multicolumn{6}{|c|}{ Table 4 Correlation matrix of IOSN component and total scores, ASA and sCMT } \\
\hline & 1 & 2 & 3 & 4 & 6 & 5 \\
\hline & MDAS & ASA & MBIS & sCMT & DTCS & Total IOSN \\
\hline 1. MDAS & 1 & 0.06 & -0.002 & 0.072 & $0.43^{* *}$ & $0.45^{* *}$ \\
\hline 2. ASA & & 1 & $0.72^{* *}$ & $0.35^{* *}$ & 0.09 & 0.16 \\
\hline 3. MBIS & & & 1 & $0.38^{* *}$ & 0.12 & $0.37^{* *}$ \\
\hline 4. SCMT & & & & 1 & 0.09 & $0.21^{*}$ \\
\hline 6. DTCS & & & & & 1 & 0.2 \\
\hline 5. IOSN & & & & & & 1 \\
\hline *P $<0.05: * * P<0.01: P<0.001^{* * *}$ & & & & & \\
\hline
\end{tabular}

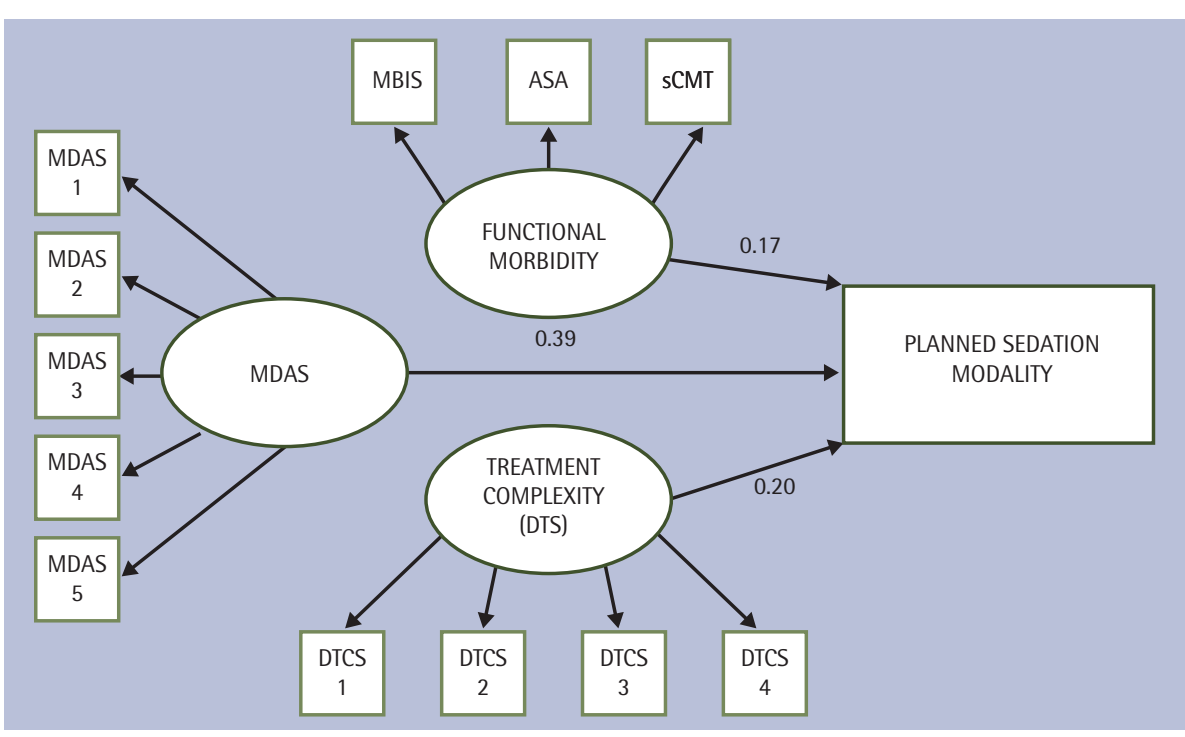

Fig. 1 Path analysis summary with standardised coefficients (error terms excluded for ease of presentation) approach and ensuring good practice and developed the IOSN and had shown its versatility in: [i] as a tool for referring patients for sedation; and [ii] as a health needs assessment tool to determine sedation need within referred patients to an oral surgery service had suggested that the IOSN had a degree of predictive and construct validity with regard to sedation modality. ${ }^{7}$ However, the question remained as to the role of the IOSN or its component parts to assist in the choice of sedation modality for patients referred for dental anxiety management to a secondary care facility. Therefore, the purpose of the work presented here, was to conduct an exploratory investigation of PDS practitioners' planned sedation modality using a structural equation modelling approach in order to identify the explanatory value of using IOSN, or its component parts, to predict sedation modality in patients referred with dental anxiety.

With regard to the IOSN, the vast majority of the patients referred for dental anxiety management to the PDS in NHS Highland, quality care. By 2011, Coulthard et al. ${ }^{2-5}$ populations. More recent work with self- 
from the General Dental Service, were categorised as having a high sedation need. Over $80 \%$ were extremely dentally anxious, required dental treatment classified by IOSN as 'intermediate' and 'complex' with over 50\% assessed as having a medical condition that could be exacerbated by dental treatment. With regard to the routine collected information, the patients with a sedation need had higher scores for dental anxiety, the planned sedation modality and normative dental treatment need. Although approaching significance the routine measures used to assess functional morbidity were less well differentiated between the sedation need groups. Nonetheless, these findings suggested that IOSN had an important role in the demarcation of sedation need in a population of highly anxious patients. Moreover, the IOSN correlated positively and significantly with the sCMT. Hence, it is tentatively suggested that with regard to construct validity, and in agreement with Liu et al., ${ }^{7}$ the IOSN performed as anticipated in that it showed good relationships with dental anxiety, case complexity, planned sedation modality and normative dental treatment need.

The path analysis showed that two components of the IOSN were predictive of the PDS practitioner's choice of planned sedation modality - that is dental anxiety (MDAS) and treatment complexity (DTC). It can be pointed out, in addition, that the strength of the parameter estimates, indicating the prediction of planned sedation modality, are at the lower bound, that is, with the dependent variable represented as a raw rating the associations have not been completely disattenuated. A speculative interpretation of the findings can be made as demonstrated from parameter estimates that populate the fitted model. That is, the dentists made their decisions on the level of sedation to provide on the basis of how dentally anxious the patient was and the normative dental treatment need (treatment complexity). The role of dental anxiety and treatment complexity and the absence of a role for functional morbidity in the prediction of sedation modality were also shown by Liu et al. ${ }^{7}$ They stated that:

'[the] Medical history did not seem to have a significant factor towards sedation need. The fact that patient anxiety and treatment complexity appear to be the more significant factor for sedation need, leads to the questioning whether the IOSN tool could be modified and/or simplified to reflect this.'

However, considering the recommendations of the 2015 Standards Report, ${ }^{1}$ we would propose that a measure of medical history assessment, as for example the ASA, must remain within the assessment of the patient referred for dental anxiety management and sedation. With regard to the present study, the lack of the functional morbidity to predict planned sedation modality may indicate that the PDS practitioners were, perhaps, more influenced by a patient-centred and treatment procedural profile and so certain components rather than the IOSN as a whole appeared to be useful in their decision-making. Therefore, it may be suggested that components of the IOSN, provided additional explanatory value with regard not only to sedation need, but also to the choice of sedation modality. In this respect the IOSN could be considered to possess utility, as a health needs assessment tool.

There are certain limitations of this study. Concerns have been raised about the use of non-probability convenience sampling associated with variability of findings, however, adopting this sampling technique is, according to Hulley et al., appropriate for initial studies which are explorative in nature. ${ }^{14}$ This path analysis, however, should be regarded as exploratory as the sample size was at the lower bound of acceptability for conducting complex modelling. However, it is considered justified as the model, when run, required a small number of iterations to fit, delivered no warnings (for example, Heywood values) and the Langrange 'modification' indices were reassuringly low. The advantage of the analysis demonstrated in a single calculation is that when controlling for the influence of the independent (that is, distal) variables, such as dental anxiety, treatment complexity etc, the direct effects on the planned sedation modality chosen were demonstrable.

The implications of this initial exploratory analysis are two-fold. Firstly, it shows that the IOSN is a useful and valid assessment of sedation need and sedation modality for patients referred with high dental anxiety states, and secondly, that component parts of the IOSN add explanatory value in practitioners' choice of planned sedation modality. The data supports the finding that the IOSN may be used to assess planned sedation need as well as acting as a referral tool as described by Coulthard et al..$^{2-6}$ To strengthen the use of the IOSN for those commissioning sedation services for patients who may require sedation, there remains a need for confirmatory studies so that commissioners and dental practitioners within primary and secondary dental services are aware of the IOSN and its role, not only in identifying unmet need for sedation, but also to assist practitioners in their decisions regarding planned sedation modality.

Acknowledgements

We would like to acknowledge the funding from the Public Dental Services, NHS Highland. We would also like to thank Sheela Tripathee for her assistance with data input and the staff and patients of NHS Highland the Public Dental Service for their cooperation.

1. The Dental Faculties of the Royal Colleagues of Surgeons and the Royal College of Anaesthetists. Standards for conscious sedation in the provision of dental care. report of the intercollegiate advisory committee for sedation in dentistry, 2015. Available online at http://www.rcseng. ac.uk/fds/publications-clinical-guidelines/docs/ standards-for-conscious-sedation-in-the-provision-of-dental-care-2015 (accessed November 2015).

2. Coulthard $P$. The indicator of sedation need (IOSN). SAAD Digest 2012; 28: 8-12.

3. Coulthard $P$, Bridgman $C M$, Gough $L$, Longman $L$, Pretty I A, Jenner T. Estimating the need for dental sedation. 1. The indicator of sedation need (IOSN) a novel assessment tool. Br Dent J 2011; 211: E10.

4. Pretty | A, Goodwin M, Coulthard P et al. Estimating the need for dental sedation. 2. Using IOSN as a health needs assessment tool. Br Dent J 2011; 211: E11.

5. Goodwin $M$, Pretty I A. Estimating the need for dental sedation. 3. Analysis of factors contributing to non-attendance for dental treatment in the general population, across 12 English primary care trusts. $\mathrm{Br}$ Dent J 2011; 211: 599-603.

6. Goodwin M, Coulthard P, Pretty I A, Bridgman C, Gough L, Sharif M O. Estimating the need for dental sedation. 4. Using IOSN as a referral tool. Br Dent J 2012; 212: E9.

7. Liu T, Pretty | A, Goodwin M. Estimating the need for dental sedation: evaluating the threshold of the IOSN tool in an adult population. Br Dent J 2013; 214: E22.

8. American Society of Anesthesiologists. ASA physical status classification system. Available online at https://www.asahq.org/resources/clinical-information/asa-physical-status-classification-system (accessed November 2015).

9. Bateman P, Arnold C, Brown R et al. BDA special care case mix model. Brit Dent J. 2010; 208: 291-296.

10. Duane B G, Humphris G M, Richards D, O'Keefe E J, Gordon K, Freeman R. Weighing up the weighted case mix tool (WCMT): a psychometric investigation using confirmatory factor analysis. Community Dent Health 2014; 31: 200-206.

11. Humphris G M, Morrison T, Lindsay S J E. The modified dental anxiety scale: UK norms and evidence for validity. Community Dent Health 1995; 12: 143-159.

12. Humphris $G M$, Crawford J R, Hill K, Gilbert $A$ Freeman R. UK Population norms for the modified dental anxiety scale with percentile calculator: Adult Dental Health Survey 2009 results. BMC Oral Health 2013; 13: 29.

13. Royal College of Dental Surgery. Standards for conscious sedation in dentistry: alternative techniques. A Report from the Standing Committee on Sedation and Dentistry. The Royal College of Anaesthetists. 2007. Available online at https://www.rcseng. ac.uk/fds/publications-clinical-guidelines/docs/ SCSDAT\%202007.pdf (accessed November 2015).

14. Hulley S B, Cummings S R, Browner W S, Grady D G, Newman T B. Chapter 3. Choosing the Study Subjects: Specification, Sampling, and Recruitment. In Designing clinical research, 4th edition. pp 27-28. Lippincott, Williams \& Wilkins: Philadelphia, 2013. 